Article

\title{
Association between Hyperosmolar Hyperglycemic State and Venous Thromboembolism in Diabetes Patients: A Nationwide Analysis in Taiwan
}

\author{
Wei-Ting Wei ${ }^{1}$, Shu-Man Lin ${ }^{2,3}{ }^{\mathbb{D}}$, Jin-Yi Hsu ${ }^{3,4}$, Ying-Ying Wu ${ }^{5}$, Ching-Hui Loh ${ }^{3,4}$ (D), Huei-Kai Huang ${ }^{3,6,7, * \mathbb{D}}$ \\ and Peter Pin-Sung Liu $4,8, *$ D
}

Citation: Wei, W.-T.; Lin, S.-M.; Hsu, J.-Y.; Wu, Y.-Y.; Loh, C.-H.; Huang,

H.-K.; Liu, P.P.-S. Association

between Hyperosmolar

Hyperglycemic State and Venous

Thromboembolism in Diabetes

Patients: A Nationwide Analysis in

Taiwan. J. Pers. Med. 2022, 12, 302.

https://doi.org/10.3390/

jpm12020302

Academic Editors: Aristotelis

Chatziioannou and Yudong Zhang

Received: 18 January 2022

Accepted: 14 February 2022

Published: 17 February 2022

Publisher's Note: MDPI stays neutral with regard to jurisdictional claims in published maps and institutional affiliations.

Copyright: (C) 2022 by the authors. Licensee MDPI, Basel, Switzerland. This article is an open access article distributed under the terms and conditions of the Creative Commons Attribution (CC BY) license (https:// creativecommons.org/licenses/by/ $4.0 /)$.
1 Department of Emergency Medicine, Hualien Tzu Chi Hospital, Buddhist Tzu Chi Medical Foundation, Hualien 970, Taiwan; timduncan1001@gmail.com

2 Department of Physical Medicine and Rehabilitation, Hualien Tzu Chi Hospital, Buddhist Tzu Chi Medical Foundation, Hualien 970, Taiwan; drshumanlin@gmail.com

3 School of Medicine, Tzu Chi University, Hualien 970, Taiwan; hsu.jinyi@gmail.com (J.-Y.H.); twdoc1960@gmail.com (C.-H.L.)

4 Center for Aging and Health, Hualien Tzu Chi Hospital, Buddhist Tzu Chi Medical Foundation, Hualien 970, Taiwan

5 Department of Psychiatry, Hualien Tzu Chi Hospital, Buddhist Tzu Chi Medical Foundation, Hualien 970, Taiwan; squirrellyying@gmail.com

6 Department of Medical Research, Hualien Tzu Chi Hospital, Buddhist Tzu Chi Medical Foundation, Hualien 970, Taiwan

7 Department of Family Medicine, Hualien Tzu Chi Hospital, Buddhist Tzu Chi Medical Foundation, Hualien 970, Taiwan

8 Institute of Medical Sciences, Tzu Chi University, Hualien 970, Taiwan

* Correspondence: drhkhuang@gmail.com (H.-K.H.); psliu520@gmail.com (P.P.-S.L.); Tel.: +886-3-8561825 (H.-K.H.); +886-3-8561825 (P.P.-S.L.); Fax: +886-3-8560977 (H.-K.H.); +886-3-8577161 (P.P.-S.L.)

Abstract: Background: Previous studies in Western countries have shown that a hyperosmolar hyperglycemic state (HHS) is associated with an increased risk of venous thromboembolism (VTE); in these cases, prophylactic anticoagulant treatment is suggested. However, the association between HHS and VTE in Asian populations remains undetermined. Therefore, we aimed to evaluate whether HHS is associated with an increased risk of VTE in diabetic Taiwanese patients. Methods: This nationwide, population-based, retrospective cohort study was conducted using the Taiwan National Health Insurance Research Database. We enrolled a total of 4,723,607 admission records of patients with diabetes diagnosed with one or more of seven common diseases (pneumonia, urinary tract infection, sepsis, heart disease, stroke, malignancy, and respiratory tract disease) between 2001 and 2018 in Taiwan. The patients were divided into two groups based on the presence $(n=46,000)$ or absence $(n=4,677,607)$ of HHS. We estimated the adjusted odds ratio (aOR) for developing VTE within 90 days after the index hospitalization using multivariable logistic regression with generalized estimating equations accounting for repeated measures. Results: Overall, patients admitted with HHS had a similar risk of VTE compared with those admitted without HHS (408/46,000 vs. $39,345 / 4,677,607 ; \mathrm{aOR}=1.06,95 \% \mathrm{CI}: 0.97-1.17, p=0.190)$. A similar non-significant association between HHS and VTE was found regardless of age and sex subgroups. Conclusions: There was no significant association between HHS and overall VTE risk in patients with diabetes in Taiwan. The results of our study may not support the use of prophylactic anticoagulant therapy in diabetic Taiwanese patients with HHS.

Keywords: hyperosmolar hyperglycemic state; venous thromboembolism; deep vein thrombosis; pulmonary embolism 


\section{Introduction}

Venous thromboembolism (VTE) is a major global health concern. This condition consists of two types: deep vein thrombosis (DVT) and pulmonary embolism (PE). The annual incidence of VTE in Asia varies from 14 to 20 per 100,000 individuals [1]. Hospitalized patients have a higher risk of VTE than non-hospitalized patients [2]. VTE can be a dangerous disease that, without early diagnosis and prompt intervention, results in a fatality rate of up to $30 \%$ [3-5].

Hyperosmolar hyperglycemic state (HHS) is a complication of diabetes mellitus (DM), characterized by severe hyperglycemia, hyperosmolality, and dehydration without significant ketoacidosis. The mortality rate of HHS can be as high as $20 \%$ [6-8]. The precipitating factors in the development of HHS include infection, discontinuation or inadequate insulin therapy, pancreatitis, myocardial infarction, cerebrovascular accident, and some specific drug use [6].

Several case reports have described VTE development during the HHS [9-12]. Severe dehydration and hyperosmolarity, resulting in the release of tissue thromboplastins from disrupted endothelial cells, may enhance coagulation [13]. In the American population, patients hospitalized for HHS were shown to have a higher risk of VTE than those with uncomplicated DM [14]. The current guidelines for the management of HHS recommend prophylactic anticoagulation for the full duration of hospitalization in all patients with HHS, unless contraindications exist [15]. However, the benefits and risks of pharmacological prophylaxis for VTE in patients with HHS have not been proven in clinical trials, Ref. [16,17] and current evidence is insufficient to support this recommendation in the Asian population. Since the risk of VTE in Asians is significantly lower than that in Westerners, [1] whether HHS increases the risk of VTE and should be treated with prophylactic anticoagulants in Asian populations is still undetermined. To date, patients with HHS or most medically ill patients do not routinely receive prophylactic anticoagulants during hospitalization in Taiwan. Therefore, this study aimed to evaluate whether HHS is associated with an increased risk of VTE among hospitalized patients with DM in Taiwan.

\section{Materials and Methods}

\subsection{Data Sources}

This nationwide retrospective cohort study was conducted using data from the National Health Insurance Database (NHID). The National Health Insurance (NHI) program was started in Taiwan in 1995 and enrolled $>99 \%$ of the Taiwanese population, approximately 23 million people, and 97\% of the hospitals and clinics [18]. The NHI of Taiwan contains abundant health and medical treatment information on insured individuals, such as inpatient and outpatient care services, emergency department services, dental care, use of traditional Chinese medicine, and other medical care [19-21]. The diagnostic codes used in the NHID conformed to the International Classification of Diseases, 9th Revision, Clinical Modification (ICD-9-CM) codes before 2016, and ICD-10-CM codes after 2016. The data of each participant was anonymized by encrypting the identification number. Patient characteristics and medical records were obtained from the Health and Welfare Data Science Center of Taiwan's Ministry of Health and Welfare. This study was conducted in accordance with the World Medical Association Declaration of Helsinki: Ethical Principles for Medical Research Involving Human Subjects. This study was approved by the Research Ethics Committee of Hualien Tzu Chi Hospital (REC No: IRB107-152-C), and the requirement for informed consent was waived.

\subsection{Study Population}

We initially identified all admissions with DM, defined as those with prescriptions of antidiabetic drugs (Anatomical Therapeutic Chemical [ATC] code: A10) during admission or within 90 days prior to admission, between 2001 and 2018 in Taiwan. To ensure the comparability between study groups, we limited the admissions to those with the main diagnoses of the following common conditions: pneumonia, urinary tract infection 
(UTI), sepsis, heart disease, stroke, malignancy, and respiratory tract disease (other than pneumonia) (Supplemental Table S1), which were found to be common diagnoses and associated with the precipitating factor of HHS among hospitalized DM patients in previous studies $[9,17]$. We divided our study population into admissions with and without the diagnosis of HHS (ICD-9: 250.2; ICD-10: E08.0, E11.0, E13.0). Patients who had been diagnosed with VTE or prescribed anticoagulants (ATC codes: B01AA, B01AB, B01AE, $\mathrm{B} 01 \mathrm{AF}$ ) within 90 days prior to admission were excluded. Patients who died within 90 days after admission without VTE diagnosis were excluded.

\subsection{Outcome Measures}

The primary outcome was defined as the occurrence of VTE within 90 days after the index date; the index date was defined as the date of admission with DM as aforementioned. The VTE included both DVT (ICD-9-CM codes: 453.2, 453.4, 453.5, 453.7, 453.8, and 453.9; ICD-10-CM codes: I82.2, I82.4, I82.5, I82.60, I82.62, I82.89, and I82.9) and PE (ICD-9-CM codes: 415.1 except for 415.11 and 415.12; ICD-10-CM codes: I26 except for I26.01 and I26.90). The accuracy of the ICD-9-CM codes for diagnosing VTE has been previously validated in Taiwan, with high positive predictive values [22]. We also evaluated the occurrence of DVT and PE as two separate secondary outcomes. In addition to the overall analysis, the comparison of VTE risk between HHS and non-HHS admissions was performed based on the subgroups of the main admission diagnoses (pneumonia, UTI, sepsis, heart disease, stroke, malignancy, and respiratory tract disease) [23].

\subsection{Covariates}

The baseline and clinical characteristics included age, sex, comorbidities, medications, and income. A pre-existing comorbidity, according to the ICD-9-CM and ICD-10-CM codes, was defined as a disease that was diagnosed during at least one hospital admission or two outpatient visits in the year prior to the index date. The Charlson Comorbidity Index was calculated to represent the overall comorbidity status [24]. Data on monthly income was retrieved to serve as socioeconomic status and was categorized into four levels ( $\geq 40,000,20,000-39,999,1-19,999$ New Taiwan dollars, or financially dependent) based on income-related NHI premiums.

\subsection{Statistical Analysis}

We used the standardized mean difference to evaluate the differences in baseline characteristics between study groups. Since each admission event was considered as a unit of analysis, some of the recorded admissions may have involved the same patient and should be considered as repeated-measure data. Therefore, we estimated the odds ratio (OR) for developing VTE using logistic regression models with generalized estimating equations accounting for the potential correlation between repeated measures within subjects. Multivariable analyses were performed with adjustment for all covariates listed in Table 1, including age, sex, income level, major diagnoses, Charlson Comorbidity Index, comorbidities, and medication use. A two-sided probability value of $<0.05$ was considered statistically significant. Data management and statistical analyses were performed using Stata, version 14 (Stata Corporation LLC; College Station, TX, USA) and SAS, version 9.4 (SAS Institute, Inc.; Cary, NC, USA). 
Table 1. General characteristics of admission records with and without HHS.

\begin{tabular}{|c|c|c|c|c|c|}
\hline \multirow{2}{*}{ Variables } & \multicolumn{2}{|c|}{$\begin{array}{c}\text { HHS } \\
(n=46,000)\end{array}$} & \multicolumn{2}{|c|}{$\begin{array}{c}\text { Non-HHS } \\
(n=4,677,607)\end{array}$} & \multirow[t]{2}{*}{ SMD } \\
\hline & $n$ & $\%$ & $n$ & $\%$ & \\
\hline$\underset{\text { Sex }}{\text { Age, mean }} \pm$ SD & \multicolumn{2}{|c|}{$70.6 \pm 13.7$} & \multicolumn{2}{|c|}{$68.7 \pm 12.9$} & 0.1481 \\
\hline Male & 23,356 & 50.8 & $2,509,758$ & 53.7 & 0.0577 \\
\hline Female & 22,644 & 49.2 & $2,167,849$ & 46.4 & 0.0577 \\
\hline \multicolumn{6}{|l|}{ Income level (NTD) } \\
\hline Dependent & 14,553 & 31.6 & $1,370,942$ & 29.3 & 0.0506 \\
\hline $15,840-24,999$ & 21,802 & 47.4 & $2,116,458$ & 45.3 & 0.0431 \\
\hline $25,000-44,999$ & 6160 & 13.4 & 773,223 & 16.5 & 0.0881 \\
\hline$\geq 45,000$ & 3485 & 7.6 & 416,984 & 8.9 & 0.0484 \\
\hline \multicolumn{6}{|l|}{ Major admission diagnosis } \\
\hline Pneumonia & 7525 & 16.4 & 507,713 & 10.9 & 0.1612 \\
\hline Urinary tract infection & 2893 & 6.3 & 198,460 & 4.2 & 0.0919 \\
\hline Sepsis & 9835 & 21.4 & 387,022 & 8.3 & 0.3754 \\
\hline Heart disease & 10,548 & 22.9 & $1,301,622$ & 27.8 & 0.1128 \\
\hline Stroke & 3349 & 7.3 & 443,937 & 9.5 & 0.0798 \\
\hline Malignancy & 2485 & 5.4 & $1,019,051$ & 21.8 & 0.4925 \\
\hline Respiratory tract disease & 9365 & 20.4 & 819,802 & 17.5 & 0.0723 \\
\hline $\begin{array}{l}\text { Charlson Comorbidity Index, } \\
\text { mean } \pm S D\end{array}$ & \multicolumn{2}{|c|}{$3.1 \pm 2.4$} & \multicolumn{2}{|c|}{$3.8 \pm 2.8$} & 0.2603 \\
\hline \multicolumn{6}{|l|}{ Comorbidities } \\
\hline Hypertension & 29,633 & 64.4 & $3,231,659$ & 69.1 & 0.0993 \\
\hline Atrial fibrillation & 2128 & 4.6 & 246,327 & 5.3 & 0.0295 \\
\hline Stroke & 14,113 & 30.7 & $1,117,407$ & 23.9 & 0.1529 \\
\hline Heart failure & 5917 & 12.9 & 688,511 & 14.7 & 0.0540 \\
\hline Coronary artery disease & 10,264 & 22.3 & $1,377,629$ & 29.5 & 0.1636 \\
\hline COPD & 7907 & 17.2 & 886,982 & 19.0 & 0.0460 \\
\hline Chronic renal failure & 5810 & 12.6 & 677,667 & 14.5 & 0.0543 \\
\hline Cirrhosis & 1942 & 4.2 & 267,805 & 5.7 & 0.0695 \\
\hline Hyperlipidemia & 10,953 & 23.8 & $1,426,584$ & 30.5 & 0.1508 \\
\hline Fracture of lower limbs & 1927 & 4.2 & 151,134 & 3.2 & 0.0508 \\
\hline Gout & 3173 & 6.9 & 381,512 & 8.2 & 0.0478 \\
\hline Malignancy & 4877 & 10.6 & $1,104,759$ & 23.6 & 0.3510 \\
\hline Thyroid dysfunction & 675 & 1.5 & 79,384 & 1.7 & 0.0184 \\
\hline \multicolumn{6}{|l|}{ Baseline medication use } \\
\hline Statins & 10,799 & 23.5 & $1,408,819$ & 30.1 & 0.1503 \\
\hline Antiplatelet & 20,027 & 43.5 & $2,202,503$ & 47.1 & 0.0714 \\
\hline
\end{tabular}

Continuous data are expressed as mean $\pm \mathrm{SD}$, and categorical data are expressed as numbers and percentages. Abbreviations: $\mathrm{COPD}=$ chronic obstructive pulmonary disease; HHS = hyperosmolar hyperglycemic state; NTD = New Taiwan Dollar; SD = standard deviation; SMD = standardized mean difference.

\section{Results}

\subsection{General Characteristics}

Overall, 4,723,607 admission records were enrolled in this study; of these, 46,000 and $4,677,607$ belonged to the HHS and non-HHS admission groups, respectively. The mean age was 70.6 years in the HHS group and 68.7 years in the non-HHS group. The top three main admission diagnoses were heart disease, sepsis, and respiratory tract disease in the HHS group and heart disease, malignancy, and respiratory tract disease in the non-HHS group. Some baseline characteristics were different between study groups, such as a higher proportion of stroke but lower proportion of coronary artery disease, hyperlipidemia, and malignancy in the HHS group than in the non-HHS group. The detailed baseline characteristics are shown in Table 1. 


\subsection{Association between HHS and VTE}

Overall, the event rates of VTE were 408/46,000 (8.87\%o) and 39,345/4,677,607 (8.41\%o) for the HHS and non-HHS groups, respectively. There was no significant association between HHS admission and the development of VTE in both univariable and multivariable analyses (crude OR = 1.06, 95\% CI: 0.97-1.17, $p=0.196$; adjusted OR [aOR] = 1.06, 95\% CI: $0.97-1.17, p=0.190$ ) (Table 2).

Table 2. Event rate and odds ratio of VTE in HHS versus non-HHS admissions.

\begin{tabular}{|c|c|c|}
\hline & HHS & Non-HHS \\
\hline Admission numbers & 46,000 & $4,677,607$ \\
\hline VTE events & 408 & 39,345 \\
\hline Event rate $(\%)$ & 8.87 & 8.41 \\
\hline \multicolumn{3}{|l|}{ Univariate model } \\
\hline Crude OR (95\% CI) & 1.06 (0.97-1.17) & 1 (ref.) \\
\hline$p$ value & 0.196 & \\
\hline \multicolumn{3}{|l|}{ Multivariable model * } \\
\hline Adjusted OR (95\% CI) & $1.06(0.97-1.17)$ & 1 (ref.) \\
\hline$p$ value & 0.190 & \\
\hline
\end{tabular}

In the age- and sex-stratified analyses, a similar non-significant association between HHS and VTE was found, regardless of age ( $<65$ years and $\geq 65$ years) and sex subgroups (Table 3). The tests for interaction also supported that no subgroup effect of age/sex existed on the association between HHS and VTE.

Table 3. Odds ratio of VTE in HHS versus non-HHS admissions stratified by age and sex.

\begin{tabular}{|c|c|c|c|c|c|}
\hline & \multicolumn{2}{|c|}{ Univariate Model } & \multicolumn{2}{|c|}{ Multivariable Model * } & \multirow[b]{2}{*}{$p$ for Interaction } \\
\hline & Crude OR $(95 \%$ CI) & $p$ Value & Adjusted OR (95\% CI) & $p$ Value & \\
\hline \multicolumn{6}{|l|}{ Age $<65$ years } \\
\hline HHSNon-HHS & $1.02(0.84-1.24)$ & 0.832 & $1.04(0.86-1.26)$ & 0.676 & \multirow{4}{*}{0.540} \\
\hline Age $\geq 65$ years & $1.00(1 \mathrm{er})$ & & 1.00 (reI) & & \\
\hline HHS & $1.08(0.98-1.20)$ & 0.138 & $1.11(1.00-1.23)$ & 0.058 & \\
\hline Non-HHS & 1.00 (ref) & & 1.00 (ref) & & \\
\hline \multicolumn{6}{|l|}{ Male } \\
\hline HHS & $1.03(0.90-1.19)$ & 0.659 & $1.05(0.91-1.21)$ & 0.484 & \multirow{5}{*}{0.670} \\
\hline Non-HHS & 1.00 (ref) & & 1.00 (ref) & & \\
\hline Female & & & & & \\
\hline HHS & $1.08(0.96-1.22)$ & 0.219 & $1.10(0.98-1.25)$ & 0.116 & \\
\hline Non-HHS & 1.00 (ref) & & 1.00 (ref) & & \\
\hline
\end{tabular}

Regarding the secondary outcomes, patients admitted with HHS were slightly more likely to develop DVT than those admitted without HHS (event rate: $6.00 \%$ o vs. 5.56\% ; $\mathrm{aOR}=1.16,95 \% \mathrm{CI}: 1.03-1.31, p=0.012)$. On the other hand, there was no significant association between HHS and PE (event rate: $3.35 \%$ vs. $3.09 \%$; aOR $=0.97,95 \% \mathrm{CI}$ : $0.83-1.12, p=0.641)$ (Table 4). 
Table 4. Event rate and odds ratio of DVT and PE in HHS versus non-HHS admissions.

\begin{tabular}{|c|c|c|c|c|c|}
\hline & \multirow[b]{2}{*}{ Event Rate (\%o) } & \multicolumn{2}{|c|}{ Univariate Model } & \multicolumn{2}{|c|}{ Multivariable Model * } \\
\hline & & $\begin{array}{c}\text { Crude OR } \\
(95 \% \text { CI })\end{array}$ & $p$ Value & $\begin{array}{l}\text { Adjusted OR } \\
(95 \% \mathrm{CI})\end{array}$ & $p$ Value \\
\hline \multicolumn{6}{|l|}{ DVT } \\
\hline HHS & 6.00 & 1.09 (0.97-1.23) & 0.137 & $1.16(1.03-1.31)$ & 0.012 \\
\hline Non-HHS & 5.56 & 1.00 (ref.) & & 1.00 (ref.) & \\
\hline \multicolumn{6}{|l|}{ PE } \\
\hline HHS & 3.35 & 1.07 (0.93-1.23) & 0.353 & $0.97(0.83-1.12)$ & 0.641 \\
\hline Non-HHS & 3.09 & 1.00 (ref.) & & 1.00 (ref.) & \\
\hline
\end{tabular}

\subsection{Analyses According to the Main Admission Diagnoses}

The association between HHS and the development of VTE was not significant in the subgroups of patients admitted due to pneumonia, sepsis, heart disease, stroke, malignancy, and respiratory tract disease (Table 5). However, we found a significant association between HHS and VTE in patients admitted for UTI (aOR = 1.70, 95\% CI: 1.19-2.51, $p=0.008)$.

Table 5. Odds ratio of VTE in HHS versus non-HHS admissions based on different admission diagnoses.

\begin{tabular}{|c|c|c|c|c|c|}
\hline \multirow[b]{2}{*}{ Main Diagnosis } & \multirow[b]{2}{*}{ Group } & \multicolumn{2}{|c|}{ Univariate Model } & \multicolumn{2}{|c|}{ Multivariable Model * } \\
\hline & & $\begin{array}{c}\text { Crude OR } \\
(95 \% \text { CI })\end{array}$ & $p$ Value & $\begin{array}{c}\text { Adjusted OR } \\
(95 \% \mathrm{CI})\end{array}$ & $p$ Value \\
\hline \multirow{2}{*}{ Pneumonia } & HHS & $1.13(0.89-1.44)$ & 0.305 & 1.17 (0.91-1.49) & 0.220 \\
\hline & Non-HHS & 1.00 (ref.) & & 1.00 (ref.) & \\
\hline \multirow{2}{*}{ Urinary tract infection } & HHS & 1.77 (1.19-2.63) & 0.005 & $1.70(1.15-2.51)$ & 0.008 \\
\hline & Non-HHS & 1.00 (ref.) & & 1.00 (ref.) & \\
\hline \multirow{2}{*}{ Sepsis } & HHS & 1.07 (0.89-1.30) & 0.465 & 1.09 (0.89-1.33) & 0.396 \\
\hline & Non-HHS & 1.00 (ref.) & & 1.00 (ref.) & \\
\hline \multirow{2}{*}{ Heart disease } & HHS & $0.95(0.76-1.18)$ & 0.625 & $0.91(0.74-1.13)$ & 0.415 \\
\hline & Non-HHS & 1.00 (ref.) & & 1.00 (ref.) & \\
\hline \multirow{2}{*}{ Stroke } & HHS & $1.49(0.99-2.24)$ & 0.058 & $1.43(0.95-2.16)$ & 0.086 \\
\hline & Non-HHS & 1.00 (ref.) & & 1.00 (ref.) & \\
\hline \multirow{2}{*}{ Malignancy } & HHS & $1.22(0.92-1.62)$ & 0.175 & $1.19(0.89-1.58)$ & 0.237 \\
\hline & Non-HHS & 1.00 (ref.) & & 1.00 (ref.) & \\
\hline \multirow{2}{*}{ Respiratory tract disease } & HHS & $0.87(0.70-1.09)$ & 0.236 & $0.88(0.70-1.10)$ & 0.264 \\
\hline & Non-HHS & 1.00 (ref.) & & 1.00 (ref.) & \\
\hline
\end{tabular}

Regarding the secondary outcomes, HHS was significantly associated with DVT development only in patients admitted for UTI ( $\mathrm{aOR}=1.95,95 \%$ CI: $1.26-3.03, p=0.003)$, but not in those admitted for the other main diagnoses (Supplementary Table S2). No significant association between HHS and PE was found in the analyses for all subgroups of the main admission diagnoses (Supplementary Table S3).

\section{Discussion}

This nationwide large-scale cohort study evaluated the association between HHS and development of VTE within 90 days after admission. We found that patients with diabetes 
hospitalized with HHS were not at a higher risk of VTE than those without HHS. This non-significant association between HHS and VTE was consistent in different age and sex subgroups. To our knowledge, this is the first Asian study using nationwide data to evaluate the association between HHS and VTE development in patients with DM.

To date, there has been limited evidence regarding the association between HHS and the risk of VTE. A previous retrospective cohort study retrieved from the California Patient Discharge Data Set showed that diabetic patients with hyperosmolarity were at an increased risk of developing VTE during their inpatient stay [14]. Moreover, the risk of VTE in HHS was higher than that in uncomplicated DM (hazard ratio [HR] = 3.0; 95\% CI: 2.1-4.5) [14]. A typical patient presenting with HHS often presents with other afflictions, such as infection, heart disease, and cerebrovascular accident; however, the aforementioned study did not explore this characteristic, which may have interfered with the results. Overall, our study results, based on the entire Taiwanese population, are inconsistent with those of the American study. Our study addressed the knowledge gap regarding the association between HHS and VTE in an Asian population.

Epidemiology data in Taiwan suggest that DM is a risk factor for developing VTE $[25,26]$. DM is regarded as a hypercoagulable state [27,28]. The exact mechanism of VTE among patients with DM is complicated and includes platelet and endothelial cell dysfunction, [29,30] coagulative activation, [31,32] and suppression of fibrinolysis [33]. Patients with HHS had more severe dehydration, which is theoretically prone to a hypercoagulable state; however, in our study, the HHS group did not have an increased risk of overall VTE compared with the non-HHS group. The results may be explained by the aggressive fluid replacement that occurs during treatment to rapidly correct the hyperosmolarity and restore intravascular volume, which corrects dehydration and may avoid a hypercoagulable state. Moreover, Asians may have more efficient fibrinolytic activity and lower hereditary thrombophilia, leading to a reduced hypercoagulable state [34-36]. Further studies are necessary to verify the exact underlying mechanisms to re-examine the proposed hypotheses. Although a statistically significant association was found between HHS and DVT risk in our secondary outcome analysis, the risk was only slightly increased (event rate: $6.00 \%$ vs. $5.56 \%$ ). This difference was not sufficient to support the use of prophylactic anticoagulant treatment in patients with HHS. Of note, the subgroup analyses incidentally showed that a significantly higher risk of VTE was found specifically in patients with HHS who were admitted with a UTI, but not in those with other main diagnoses (pneumonia, sepsis, heart disease, stroke, malignancy, or respiratory tract disease). The exact underlying mechanism of this finding is still unknown, and further studies are still needed to illustrate the association between HHS and VTE in patients admitted for UTI.

Although further evidence is still needed to strengthen our findings, they hold significant implications. Previous studies have revealed that Asians have a significantly lower risk of developing VTE than Western populations [1,37,38]. Therefore, the association between HHS and VTE derived from Western populations is unlikely to be directly applicable to Asian groups. Prophylactic anticoagulation was suggested for HHS hospitalization in Western countries, where the benefit and bleeding risk may not be the same as those found in Asia. Although some conditions (such as malignancy, pneumonia, and heart failure) are considered risk factors for VTE, prophylactic anticoagulants are not routinely prescribed in Taiwan [39-42]. Our study further supported that, unlike in Western populations, prophylactic anticoagulants seem unnecessary for patients with HHS in Taiwan. Although a slightly elevated DVT risk may be observed in patients with HHS, clinicians should weigh the pros and cons (e.g., risk of major bleeding) of anticoagulant treatment. Our study findings may not support the routine use of prophylactic anticoagulant therapy for patients with HHS in Taiwan [43].

The main advantage of the present study was the large sample size included in this nationwide population-based design. However, this study has some limitations. First, some clinical data, such as patient lifestyle, cognition level, physical function, body mass index, and laboratory test results, could not be collected; thus, unmeasured factors may 
have confounded the results. Second, personal identifiers were encrypted by the Health and Welfare Data Science Center in Taiwan. Indeed, we only obtained anonymous data and were unable to confirm diagnostic accuracy by visiting patients directly, but instead relied on claims-based data. However, the diagnostic accuracy of VTE, the main study outcome, was proven to have high positive predictive values in Taiwan [22]. Third, although the diagnoses and treatment of DM, as well as other comorbidities, may have changed over time, our analyses did not consider the potential differences over varying periods. Further studies are required to address this issue. Fourth, this study was conducted in Taiwan and its generalizability to other Asian countries is still questionable.

In conclusion, this nationwide large-scale cohort study demonstrated that there was no significant association between HHS and overall VTE risk among patients with DM in Taiwan. Although a slightly higher risk of DVT was found in patients with HHS, the magnitude of the increase was small. Our study may, thus, not support the use of prophylactic anticoagulant therapy in Taiwanese diabetic patients with HHS. Further research is warranted to confirm this finding for the development of evidence-based guidelines.

Supplementary Materials: The following are available online at https:/ /www.mdpi.com/article/10 .3390/jpm12020302/s1, Table S1: Main admission diagnosis codes, Table S2: Odds ratio of DVT in HHS versus non-HHS admissions based on different main admission diagnoses, Table S3: Odds ratio of PE in HHS versus non-HHS admissions based on different main admission diagnoses.

Author Contributions: The study was conceived and designed by W.-T.W., H.-K.H. and P.P.-S.L. Data collection was conducted by S.-M.L., J.-Y.H., H.-K.H. and P.P.-S.L. Analysis and interpretation of data were conducted by W.-T.W., H.-K.H. and P.P.-S.L. The manuscript was drafted by W.-T.W. and H.-K.H. Critical revisions were provided by W.-T.W., S.-M.L., J.-Y.H., Y.-Y.W., C.-H.L., H.-K.H. and P.P.-S.L. All authors have read and agreed to the published version of the manuscript.

Funding: This study was supported by a grant from the Hualien Tzu Chi Hospital (no. TCRD 109-33). The funder had no role in the design and conduction of the study or in the decision to approve and submit the manuscript for publication.

Institutional Review Board Statement: This study was approved by the Research Ethics Committee of Hualien Tzu Chi Hospital (REC No: IRB107-152-C), approval date: 14 August 2018.

Informed Consent Statement: The Research Ethics Committee of Hualien Tzu Chi Hospital approved this study, and the requirement for informed consent was waived due to anonymized data.

Data Availability Statement: The dataset used in this study is managed by the Taiwan Ministry of Health and Welfare and thus cannot be made available publicly. Researchers interested in accessing this dataset can submit a formal application to the Ministry of Health and Welfare to request access (Taiwan Ministry of Health and Welfare, No. 488, Section 6, Zhongxiao E Rd, Nangang District, Taipei 115, Taiwan; website: https://dep.mohw.gov.tw/DOS/cp-2516-59203-113.html. The last accessing date: 2 February 2022).

Acknowledgments: The authors would like to thank the Health and Welfare Data Science Center, Ministry of Health and Welfare, Taiwan for approving our access to the database, and the Health and Welfare Data Science Center of Tzu Chi University for facilitating data extraction.

Conflicts of Interest: The authors declare that no conflict of interest exist.

\section{References}

1. Lee, L.H.; Gallus, A.; Jindal, R.; Wang, C.; Wu, C.C. Incidence of Venous Thromboembolism in Asian Populations: A Systematic Review. Thromb. Haemost. 2017, 117, 2243-2260. [CrossRef] [PubMed]

2. Heit, J.A.; Silverstein, M.D.; Mohr, D.N.; Petterson, T.M.; O'Fallon, W.M.; Melton, L.J. Risk factors for deep vein thrombosis and pulmonary embolism: A population-based case-control study. Arch. Intern. Med. 2000, 160, 809-815. [CrossRef] [PubMed]

3. Cushman, M.; Tsai, A.W.; White, R.H.; Heckbert, S.R.; Rosamond, W.D.; Enright, P.; Folsom, A.R. Deep vein thrombosis and pulmonary embolism in two cohorts: The longitudinal investigation of thromboembolism etiology. Am. J. Med. 2004, 117, 19-25. [CrossRef] 
4. Bĕlohlávek, J.; Dytrych, V.; Linhart, A. Pulmonary embolism, part I: Epidemiology, risk factors and risk stratification, pathophysiology, clinical presentation, diagnosis and nonthrombotic pulmonary embolism. Exp. Clin. Cardiol. 2013, 18, 129-138. [PubMed]

5. Burgazli, K.M.; Atmaca, N.; Mericliler, M.; Parahuleva, M.; Erdogan, A.; Daebritz, S.H. Deep vein thrombosis and novel oral anticoagulants: A clinical review. Eur. Rev. Med. Pharmacol. Sci. 2013, 17, 3123-3131.

6. Kitabchi, A.E.; Umpierrez, G.E.; Miles, J.M.; Fisher, J.N. Hyperglycemic crises in adult patients with diabetes. Diabetes Care 2009, 32, 1335-1343. [CrossRef]

7. Fadini, G.P.; de Kreutzenberg, S.V.; Rigato, M.; Brocco, S.; Marchesan, M.; Tiengo, A.; Avogaro, A. Characteristics and outcomes of the hyperglycemic hyperosmolar non-ketotic syndrome in a cohort of 51 consecutive cases at a single center. Diabetes Res. Clin. Pract. 2011, 94, 172-179. [CrossRef] [PubMed]

8. Milionis, H.J.; Elisaf, M.S. Therapeutic management of hyperglycaemic hyperosmolar syndrome. Expert Opin. Pharmacother. 2005, 6, 1841-1849. [CrossRef]

9. Whelton, M.J.; Walde, D.; Havard, C.W. Hyperosmolar non-ketotic diabetic coma: With particular reference to vascular complications. Br. Med. J. 1971, 1, 85-86. [CrossRef]

10. Siah, S.; Benameur, B.; Dimou, M. Hyperosmolar coma and cerebral thrombophlebitis. Ann. Fr. Anesth. Reanim. 2002, 21, 540-541. [CrossRef]

11. Scharf, Y.; Nahir, M.; Tatarsky, I.; Arieh, J.B.; Baruch, G.; Edelman, S. Fatal venous thrombosis in hyperosmolar coma. Diabetes 1971, 20, 308-309. [CrossRef] [PubMed]

12. Park, J.; Kim, D.J.; Kim, H.Y.; Seo, J.A.; Kim, S.G.; Baik, S.H.; Choi, D.S. Extensive venous thrombosis of the upper extremity in a diabetic patient with a hyperosmolar hyperglycemic state. Korean J. Intern. Med. 2006, 21, 244-247. [CrossRef]

13. Grant, P.J.; Tate, G.M.; Hughes, J.R.; Davies, J.A.; Prentice, C.R.M. Does hypernatraemia promote thrombosis? Thromb. Res. 1985, 40, 393-399. [CrossRef]

14. Keenan, C.R.; Murin, S.; White, R.H. High risk for venous thromboembolism in diabetics with hyperosmolar state: Comparison with other acute medical illnesses. J. Thromb. Haemost. 2007, 5, 1185-1190. [CrossRef] [PubMed]

15. Scott, A.R. Management of hyperosmolar hyperglycaemic state in adults with diabetes. Diabet. Med. 2015, 32, 714-724. [CrossRef]

16. Stoner, G.D. Hyperosmolar Hyperglycemic State. Am. Fam. Physician 2017, 96, 729-736.

17. Pasquel, F.J.; Umpierrez, G.E. Hyperosmolar hyperglycemic state: A historic review of the clinical presentation, diagnosis, and treatment. Diabetes Care 2014, 37, 3124-3131. [CrossRef]

18. Ho Chan, W.S. Taiwan's healthcare report 2010. EPMA J. 2010, 1, 563-585. [CrossRef] [PubMed]

19. Wu, T.Y.; Majeed, A.; Kuo, K.N. An overview of the healthcare system in Taiwan. Lond. J. Prim. Care 2010, 3, 115-119. [CrossRef]

20. Lu, J.F.; Hsiao, W.C. Does universal health insurance make health care unaffordable? Lessons from Taiwan. Health Aff. (Proj. Hope) 2003, 22, 77-88. [CrossRef]

21. Cheng, T.-M. Lessons from Taiwan's universal national health insurance: A conversation with Taiwan's Health Minister ChingChuan Yeh. Health Aff. 2009, 28, 1035-1044. [CrossRef]

22. Ng, K.J.; Lee, Y.K.; Huang, M.Y.; Hsu, C.Y.; Su, Y.C. Risks of venous thromboembolism in patients with liver cirrhosis: A nationwide cohort study in Taiwan. J. Thromb. Haemost. 2015, 13, 206-213. [CrossRef]

23. Wang, J.S.; Wu, Y.L.; Shin, S.J.; Tien, K.J.; Chin, M.C.; Hwu, C.M. Hospitalization in patients with type 2 diabetes mellitus in Taiwan: A nationwide population-based observational study. J. Formos. Med. Assoc. 2019, 118 (Suppl. 2), S90-S95. [CrossRef]

24. Charlson, M.E.; Pompei, P.; Ales, K.L.; MacKenzie, C.R. A new method of classifying prognostic comorbidity in longitudinal studies: Development and validation. J. Chronic Dis. 1987, 40, 373-383. [CrossRef]

25. Peng, Y.H.; Lin, Y.S.; Chen, C.H.; Tsai, K.Y.; Hung, Y.C.; Chen, H.J.; Chen, H.J.; Liao, W.C.; Ho, W.C.; Islam, M.A. Type 1 diabetes is associated with an increased risk of venous thromboembolism: A retrospective population-based cohort study. PLOS ONE 2020 15, e226997. [CrossRef]

26. Chung, W.S.; Lin, C.L.; Kao, C.H. Diabetes increases the risk of deep-vein thrombosis and pulmonary embolism. A populationbased cohort study. Thromb. Haemost. 2015, 114, 812-818. [CrossRef] [PubMed]

27. Carr, M.E. Diabetes mellitus: A hypercoagulable state. J. Diabetes Complicat. 2001, 15, 44-54. [CrossRef]

28. Lemkes, B.A.; Hermanides, J.; DeVries, J.H.; Holleman, F.; Meijers, J.C.; Hoekstra, J.B. Hyperglycemia: A prothrombotic factor? J. Thromb. Haemost. 2010, 8, 1663-1669. [CrossRef] [PubMed]

29. Grant, P.J. Diabetes mellitus as a prothrombotic condition. J. Intern. Med. 2007, 262, 157-172. [CrossRef] [PubMed]

30. Rollini, F.; Franchi, F.; Muniz-Lozano, A.; Angiolillo, D.J. Platelet function profiles in patients with diabetes mellitus. J. Cardiovasc. Transl. Res. 2013, 6, 329-345. [CrossRef] [PubMed]

31. Ferreiro, J.L.; Gómez-Hospital, J.A.; Angiolillo, D.J. Platelet abnormalities in diabetes mellitus. Diabetes Vasc. Dis. Res. 2010, 7 , 251-259. [CrossRef]

32. Avogaro, A.; Albiero, M.; Menegazzo, L.; de Kreutzenberg, S.; Fadini, G.P. Endothelial dysfunction in diabetes: The role of reparatory mechanisms. Diabetes Care 2011, 34 (Suppl. 2), S285-S290. [CrossRef] [PubMed]

33. Hess, K.; Grant, P.J. Inflammation and thrombosis in diabetes. Thromb. Haemost. 2011, 105 (Suppl. 1), S43-S54. [CrossRef] [PubMed]

34. White, R.H. The epidemiology of venous thromboembolism. Circulation 2003, 107, I4-I8. [CrossRef] 
35. Roberts, L.N.; Patel, R.K.; Arya, R. Venous thromboembolism and ethnicity. Br. J. Haematol. 2009, 146, 369-383. [CrossRef] [PubMed]

36. White, R.H.; Keenan, C.R. Effects of race and ethnicity on the incidence of venous thromboembolism. Thromb. Res. 2009, 123 (Suppl. 4), S11-S17. [CrossRef]

37. Anonymous. Thrombosis: A major contributor to the global disease burden. J. Thromb. Haemost. 2014, 12, 1580-1590. [CrossRef]

38. Klatsky, A.L.; Armstrong, M.A.; Poggi, J. Risk of pulmonary embolism and/or deep venous thrombosis in Asian-Americans. Am. J. Cardiol. 2000, 85, 1334-1337. [CrossRef]

39. Lee, L.H.; Nagarajan, C.; Tan, C.W.; Ng, H.J. Epidemiology of Cancer-Associated Thrombosis in Asia: A Systematic Review. Front. Cardiovasc. Med. 2021, 8, 669288. [CrossRef]

40. Tang, L.; Wu, Y.Y.; Lip, G.Y.; Yin, P.; Hu, Y. Heart failure and risk of venous thromboembolism: A systematic review and meta-analysis. Lancet Haematol. 2016, 3, e30-e44. [CrossRef]

41. Chen, C.Y.; Liao, K.M. The Incidence of Deep Vein Thrombosis in Asian Patients With Chronic Obstructive Pulmonary Disease. Medicine 2015, 94, e1741. [CrossRef] [PubMed]

42. Chen, Y.G.; Lin, T.Y.; Huang, W.Y.; Lin, C.L.; Dai, M.S.; Kao, C.H. Association between pneumococcal pneumonia and venous thromboembolism in hospitalized patients: A nationwide population-based study. Respirology 2015, 20, 799-804. [CrossRef] [PubMed]

43. Levine, M.N.; Raskob, G.; Landefeld, S.; Kearon, C. Hemorrhagic complications of anticoagulant treatment. Chest 1998, 114, 511s-523s. [CrossRef] [PubMed] 